\title{
Can fish oil supplementation improve endothelial function in asymptomatic offspring of patients with peripheral arterial disease?
}

This article was published in the following Dove Press journal:

Open Access Journal of Clinical Trials

10 July 2013

Number of times this article has been viewed

\author{
J lan Spark' \\ Christopher L Delaney' \\ Richard B Allan' \\ Melissa $\mathrm{HL} \mathrm{Ho}^{2}$ \\ Michelle D Miller ${ }^{2}$ \\ 'Department of Vascular Surgery, \\ Flinders Medical Centre and Flinders \\ University, ${ }^{2}$ Department \\ of Nutrition and Dietetics, Flinders \\ University, Bedford Park, Adelaide, \\ South Australia, Australia
}

Background: Peripheral arterial disease affects $10 \%-25 \%$ of adults aged $>55$ years, and while a multitude of risk factors exist, one key influence is genetics. Rather than awaiting the onset of debilitating symptoms, interventions that target high-risk individuals and prevent or delay the onset of symptoms would have widespread impact. The aim of this study is to implement a 12 -week fish oil intervention $(10 \mathrm{~mL} /$ day containing approximately $1.5 \mathrm{~g}$ of eicosapentaenoic acid and $1 \mathrm{~g}$ of docosahexaenoic acid), with the intention of improving endothelial function, inflammation, and lipid status in a high-risk population, ie, those with impaired endothelial function and a parent with symptomatic peripheral arterial disease.

Methods: This is a parallel-group, double-blind, randomized controlled trial involving administration of fish oil containing either about $1.5 \mathrm{~g}$ of docosahexaenoic acid and $1 \mathrm{~g}$ of docosahexaenoic acid (intervention) or about $0.15 \mathrm{~g}$ of eicosapentaenoic acid and about $0.1 \mathrm{~g}$ of docosahexaenoic acid for 12 consecutive weeks (control). The participants are 100 offspring of adults with diagnosed peripheral arterial disease who themselves have an ankle-brachial pressure index $\geq 0.9$ but impaired endothelial function according to peripheral arterial tonometry. Measures performed at baseline and at 6 and 12 weeks include flow-mediated dilatation, C-reactive protein, absolute neutrophil and lymphocyte counts, tumor necrosis factor- $\alpha$, interleukin- $1 \beta$, and interleukin- 6 levels, thromboxane and prostacyclin, lipid status, and homocysteine, nitrite, and nitrate levels. Participants will be phoned fortnightly to monitor adherence and side effects, while participants will maintain a diary of fish oil consumption on a daily basis, and fish oil returned will be measured to confirm adherence. Participants will complete validated surveys to determine background diet and physical activity levels.

Discussion: This study will examine the effectiveness of a moderate-dose fish oil intervention in reversing endothelial dysfunction in asymptomatic offspring of patients with peripheral arterial disease. It provides the opportunity to delay the progression of peripheral arterial disease using a cheap and readily available dietary supplement that has minimal side effects compared with synthetic vasoactive pharmacological medications.

Keywords: omega 3 fatty acids, endothelial function, peripheral arterial disease

\section{Background}

Peripheral arterial disease (PAD) is a major public health problem in Australia. The incidence increases with age, and an estimated $10 \%-25 \%$ of men and women over the age of 55 years are affected. ${ }^{1}$ The direct health care cost of PAD in Australia was $\$ 180$ million in 1994, of which $78 \%$ was associated with hospitalizations. ${ }^{2,3}$ PAD is also a marker for advanced cardiovascular disease involving coronary, cerebral, renal, and aortic vessels, with a 2-3-fold increased risk of cardiovascular disease-related mortality. ${ }^{4,5}$ In 2006-2007, 25,813 hospitalizations and 2163 deaths were a result
Correspondence: J lan Spark

Department of Vascular Surgery, Flinders Medical Centre, Flinders Drive, Bedford

Park, Adelaide, SA 5042, Australia

Tel +61882045445

Fax +61882047106

Email ian.spark@health.sa.gov.au
Open Access Journal of Clinical Trials 2013:5 83-91

(c) 2013 Spark et al, publisher and licensee Dove Medical Press Ltd. This is an Open Access article which permits unrestricted noncommercial use, provided the original work is properly cited. 
of PAD. ${ }^{6}$ The aging population will see an increase in the prevalence of PAD and consequently the national annual health expenditure on cardiovascular disease is likely to increase, greatly exceeding the 5.4 billion dollars spent in 2000-2001 in Australia alone. ${ }^{7}$

\section{Endothelial dysfunction and its link with PAD}

The vascular endothelium is the largest endocrine organ in the body. Its nonthrombogenic surface and ability to act as a selective barrier between the vessel lumen and surrounding tissues enable intrinsic control over inflammation, coagulation, and vasomotor tone. ${ }^{8-10}$ Loss of this ability is defined as endothelial dysfunction, and is characterized by an impaired endothelium-dependent vasodilatory response induced by changes in flow or stimuli. ${ }^{11}$ Endothelial dysfunction is associated with a systemic proinflammatory state as evidenced by increased levels of inflammatory cytokines, including interleukin-6, interleukin-1 $\beta$, and tumor necrosis factor- $\alpha$. It is also strongly predictive of future cardiovascular events, ${ }^{8,12,13}$ and is contributed to by established vascular risk factors, including smoking, hypertension, diabetes, hypercholesterolemia, and obesity. For this reason, its onset could be considered a sentinel event in the progression of vascular disease and onset of atherosclerosis. It is widely accepted that PAD is preceded by endothelial dysfunction. ${ }^{10,12}$ Significantly, endothelial dysfunction is reversible and has been shown to respond well to various treatments, particularly those modifying risk factors, such as statins and antihypertensive agents, which have vasoactive properties. ${ }^{14,15}$

\section{Assessment of endothelial function}

Flow-mediated dilatation is a surrogate marker of endothelial function and is based on the premise that blood flow creates shear stress, a physiological stimulus resulting in mechanical deformation of the endothelium and triggering release of vasodilatory substances, including nitric oxide, prostaglandins, and endothelium-derived hyperpolarizing factor. ${ }^{15}$ These diffuse to the smooth muscle cells and subsequently lead to increased vessel luminal diameter to an extent dependent on the functional ability of the endothelium. ${ }^{16}$

While assessment of endothelial function using intravascular administration of acetylcholine as a stimulus is considered the gold standard, ${ }^{17}$ this technique is invasive and not easily accessible. More commonly, a transient increase in blood flow/shear stress elicited by ischemia-induced hyperemia enables noninvasive quantification of flow-mediated dilatation using ultrasound technology. The brachial artery is typically used as the conduit vessel to assess this, and impaired vasodilatory responses are associated with most forms of cardiovascular disease, such as hypertension, coronary artery disease, chronic heart failure, PAD, diabetes, and chronic renal failure. ${ }^{18}$

While flow-mediated dilatation using the brachial artery can provide real-time images of the response to ischemiainduced hyperemia, it requires a trained sonographer and is therefore highly user-dependent in both data acquisition and analysis. It is widely used to assess pharmacologic and physiologic interventions but cannot be used for initial risk stratification because there are no agreed values for application of this technique.

Recently, a method of screening for endothelial dysfunction has been developed using peripheral arterial tonometry. This is an operator-independent, noninvasive technique that measures the arterial pulse wave amplitude using pneumatic fingertip probes during ischemia-induced hyperemia, thus allowing a reactive hyperemic index to be calculated. It enables endothelial health to be categorized as either dysfunctional, high risk for progression to dysfunction, or low risk. ${ }^{19}$ The hyperemic index has been shown to correlate closely with assessment of coronary endothelial function using invasive acetylcholine challenge during angiography. ${ }^{20}$

\section{Heritable basis of PAD}

The possible existence of an inherited genetic predisposition to PAD has been investigated in various types of familial aggregation and genetic association studies, with inconclusive results. ${ }^{21}$ This is not surprising, considering that PAD is more common in older populations and recruitment for such family-based studies is challenging. Despite this, there is evidence linking several genomic variants with increased susceptibility to PAD. ${ }^{21}$ This raises the possibility that endothelial dysfunction, a known precipitant of atherosclerosis and subsequently PAD, may manifest itself early in asymptomatic offspring of patients with PAD. Such a theory is supported by an unpublished study in our unit demonstrating that more than $50 \%$ of asymptomatic offspring of patients with symptomatic PAD have endothelial dysfunction.

\section{Benefits of fish oil in vascular disease}

Patients with PAD show an increase in plasma markers of inflammation and also failing of the circulation in affected limbs. ${ }^{22}$ Eicosapentaenoic acid (EPA) and docosahexaenoic acid (DHA) are the major polyunsaturated fatty acids in fish oil. They have been demonstrated to exert anti-inflammatory effects within the vessel wall by competing with arachidonic 
acid for membrane phospholipid insertion, thus reducing the amount of potent inflammatory eicosanoids and cytokines being produced..$^{22}$ One manifestation of this is an increased prostacyclin/thromboxane ratio, which can exert antithrombotic effects by reducing the aggregation of erythrocytes, thus increasing the flow of blood within vessels. ${ }^{22}$

Dietary supplementation with fish oil rich in n-3 polyunsaturated fatty acids has been shown to impact positively on vascular function in the setting of atherosclerosis. ${ }^{23}$ Despite this, little is known about whether improvements in endothelial function can be achieved in asymptomatic offspring of those with PAD using n-3 polyunsaturated fatty acids. Early detection and intervention using a noninvasive, readily available, affordable, and acceptable supplement such as fish oil has the potential to improve clinical management and prevent or delay disease onset in those at high risk of developing symptomatic PAD.

\section{Materials and methods Selection of participants}

The diagnosis of PAD can be made using the ankle-brachial pressure index. This is calculated by dividing the systolic blood pressure at the ankles by the systolic blood pressure in the arms. It is a simple and noninvasive method for detecting PAD. Patients with an ankle-brachial pressure index $<0.9$ are diagnosed as having PAD.

All offspring from patients with a confirmed diagnosis of PAD (ankle-brachial pressure index $<0.9$ ) who attend the department of vascular surgery at Flinders Medical Centre (Adelaide, South Australia) will be eligible for screening of endothelial function until a total of 100 participants are enrolled in the study.

The study protocol has been approved by the Southern Adelaide clinical human research ethics committee (registration number EC00188). This committee operates in accordance with the National Statement on Ethical Conduct in Human Research of 2007. An overview of the study protocol is provided in Figure 1.

\section{Inclusion and exclusion criteria}

To be included in the study, participants must have a parent with PAD, be aged 18 years or older, have a reactive hyperemiaperipheral artery tonometry index $<2.07$ (indicating high risk of progression to endothelial dysfunction) $)^{19}$ and have no evidence of established PAD (ankle-brachial pressure index $\geq 0.9$ ). ${ }^{24}$ Patients will be excluded if they ingest $2 \mathrm{~g}$ or more of total omega-3 daily as per the Fish Food Frequency Questionnaire, ${ }^{25}$ have a seafood allergy, a life expectancy less than 12 weeks, are unable to communicate effectively to provide informed consent, are receiving treatment doses of any antithrombotic agent or dual antiplatelet agents (such as aspirin plus clopidogrel, prasugrel, or ticlopidine), or are diagnosed with a bleeding disorder such as von Willebrand disease or hemophilia. Criteria 5-7 have been included due to the antithrombotic properties of fish oil and a potentially increased risk of bleeding.

\section{Study design}

The study is a double-blind, randomized, controlled trial. Participants will be randomly allocated to one of two groups, ie, an intervention group receiving 12 weeks of treatment with $10 \mathrm{~mL}$ of fish oil daily (containing about $1.5 \mathrm{~g}$ EPA and $1 \mathrm{~g}$ DHA) or a control group receiving 12 weeks of treatment with $10 \mathrm{~mL}$ of fish oil daily containing about $0.15 \mathrm{~g}$ EPA and $0.1 \mathrm{~g}$ DHA.

Allocation to these groups will be managed by the Flinders University statistician who provides a randomization service using computerized software. Allocation will be stratified by fish intake (more than two oily fish meals per week or two or less oily fish meals per week), presence of type 1 or 2 diabetes mellitus, and according to whether the participant is taking vasoactive medication or not. Research staff assessing participant outcomes will be masked to allocation.

\section{Fish oil supplementation}

For maximum adherence and minimal cost, administration of EPA and DHA will be via nonencapsulated fish oil taken with fruit or vegetable juice. Participants will be required to float $10 \mathrm{~mL}$ of fish oil (Melrose Omega 18/12 fish oil, AustL 93186) on 30-40 mL of juice (or any other flavored still drink) daily. If this dosage is not tolerated, participants can consume $5 \mathrm{~mL}$ of fish oil twice per day or otherwise consume fish oil capsules (total of eight per day, AustL 156237). The fish oil concentrate (about $1.5 \mathrm{~g}$ EPA and $1 \mathrm{~g}$ DHA per $10 \mathrm{~mL}$ ) selected for the intervention group is flavored with lemon and lime oil to mask the fish scent and taste. The fish oil selected for the control group (about $0.15 \mathrm{~g}$ EPA and $0.1 \mathrm{~g}$ DHA per $10 \mathrm{~mL}$ ) has been prepared commercially by Melrose to contain $10 \%$ of the active dose, and is also flavored with lemon and lime. To avoid any side effects associated with consumption of fish oil, the participants will consume the juice-fish mix with breakfast to avoid consumption on an empty stomach. Research staff will provide all participants with mentoring and support regarding appropriate administration to ensure minimal side effects. Nonencapsulated fish oil has been chosen because it is in widespread use and 


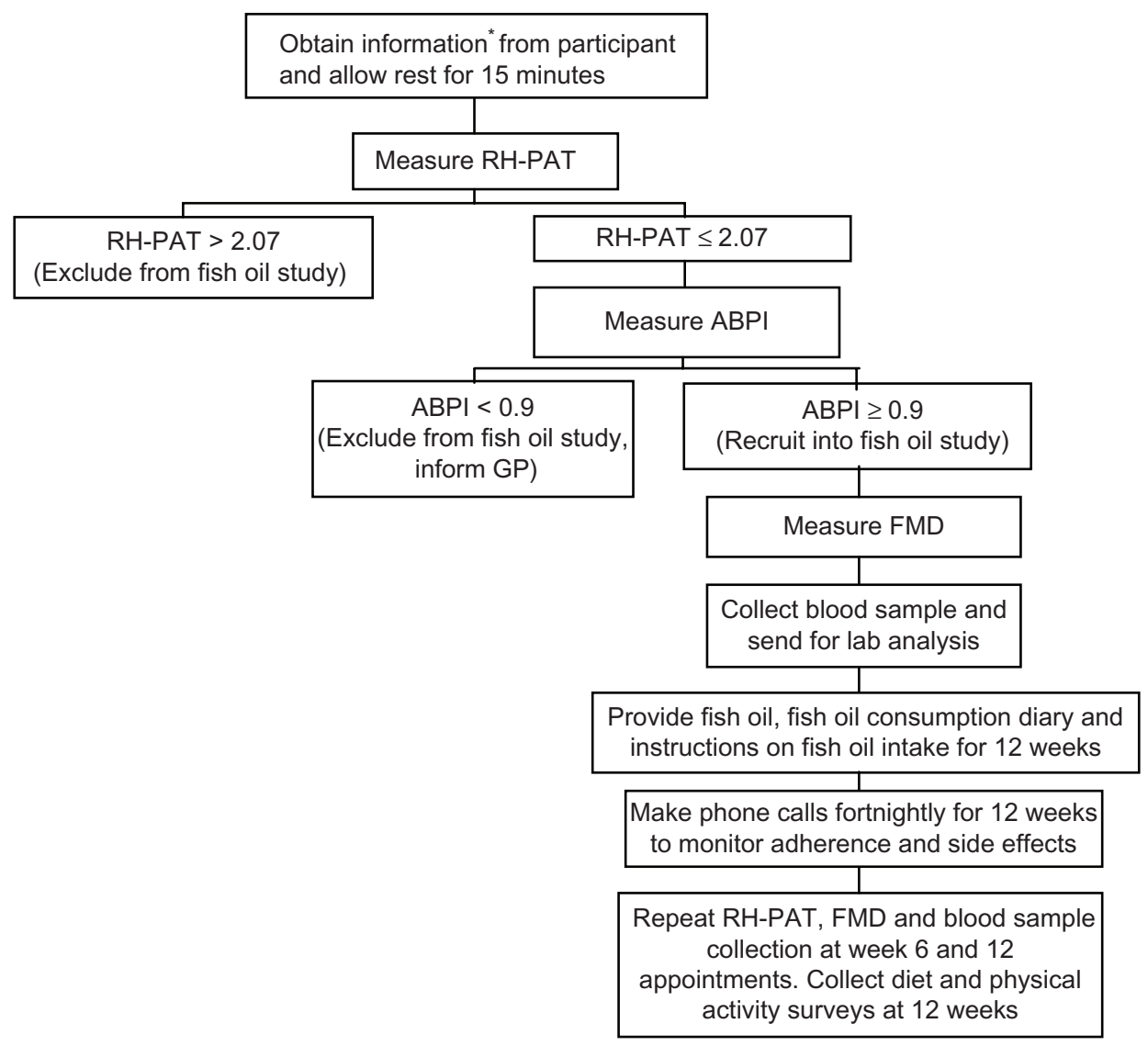

Figure I Measurements for screening and at baseline, and outcome assessments at weeks 6 and I2

Note: *Information obtained includes height, weight, blood pressure, currently taken medications, and known medical conditions of diabetes mellitus, high blood pressure, and hyperlipidemia.

Abbreviations: RH-PAT, reactive hyperemia peripheral arterial tonometry; ABPI, ankle brachial pressure index; FMD, flow mediated dilatation.

the dose considered to give therapeutic effects can be taken more simply than the alternative (about eight capsules are required to provide similar amounts of EPA as liquid fish oil). Intervention for 12 weeks has been selected because previous studies suggest that this may be sufficient to observe improvement in vascular function. ${ }^{26}$

The $10 \%$ fish oil and flavoring with citrus for the control group will assist in maintaining participant blinding to allocation. It is extremely unlikely that this low dose of fish oil provided to the control group will alter outcomes because large doses are required to provide any physiological effect. $^{27}$

To monitor adherence with the fish oil, all participants will be provided with a diary to record the daily volume consumed. To support participants, fortnightly phone calls will be made to troubleshoot any issues with compliance. Staff will also administer a series of questions designed to determine the frequency and severity of side effects and monitor changes in medications. Participants will be asked to return any unused fish oil at the 6-week and 12-week outcome assessments to confirm compliance.

\section{Endothelial function and ankle-brachial pressure index}

Endothelium-mediated changes in vascular tone will be quantified by reactive hyperemia-peripheral artery tonometry index using an EndoPAT device (Itamar Medical Ltd, Caesarea, Israel), and confirmed by flow-mediated dilatation using high resolution ultrasound. This will be done at baseline, and at six and 12 weeks following commencement of treatment.

Subject preparation will be consistent with recently published guidelines for performing flow-mediated dilatation. ${ }^{15}$ Subjects will be requested to fast for at least 8 hours beforehand (except for the consumption of water), to refrain from caffeine, alcohol, and tobacco consumption for at least 8 hours prior and to avoid strenuous exercise for 8 hours prior. Patients will wait in a quiet, temperature-controlled room for 20 minutes prior to the test. 


\section{Reactive hyperemia-peripheral artery tonometry technique}

Reactive hyperemia-peripheral artery tonometry will be performed using the EndoPAT device. The subject's blood pressure will be obtained using the right arm with a standard blood pressure cuff and sphygmomanometer; the blood pressure cuff will then be placed, but not inflated, on the left upper arm. After an explanation of the test procedure, the patient will be seated comfortably at a desk and noninvasive pneumatic probes will be attached according to the manufacturer's instructions. This will usually be on bilateral index fingers unless the patient's fingers are too large for the probes, in which case a smaller finger will be used. The patient will face away from the investigators and the EndoPAT computer display. The investigators will refrain from talking to the subject during the test except for the brief instructions required during the test. The EndoPAT device will continuously record digital arterial pressures for the duration of the test, ie, a total of 15 minutes. A five-minute baseline period prior to occlusion will be followed by five minutes of total brachial artery occlusion achieved with the cuff inflated to $250 \mathrm{mmHg}$, followed by a five-minute post occlusion period to measure the degree of reactive hyperemia. The hyperemic index will be measured using proprietary software in the EndoPAT device. Subjects with a hyperemic index of $<2.07$ will be eligible to continue in the study. According to the manufacturer of the EndoPAT, patients with a hyperemic index of $<1.67$ are deemed to have endothelial dysfunction, while subjects with a hyperemic index of 1.67-2.07 are within a sensitive zone predisposing to future development of endothelial dysfunction. It is deemed relevant to include those in the sensitive zone, given the target population of adult children at high risk of developing vascular disease as a result of family history.

\section{Ankle-brachial pressure index technique}

Subjects will then have an ankle-brachial pressure index test to ascertain whether there is evidence of established PAD. This will be obtained with the subject lying supine on a bed using the standard Doppler pressure technique. ${ }^{24} \mathrm{~A}$ blood pressure cuff, sphygmomanometer, and continuous wave Doppler (Vista ABI L450VA, Summit Doppler, Golden, CO, USA) will be used to measure the systolic blood pressure (in $\mathrm{mmHg}$ ) in both brachial arteries and in the posterior tibial and dorsalis pedis arteries in both ankles. The ankle-brachial pressure index for each ankle artery will be the systolic blood pressure of that artery divided by the higher of the two brachial artery pressures. Subjects with an ankle-brachial pressure index $<0.9$ in any ankle artery will be considered to have evidence of established PAD and will be excluded from the study.

\section{Flow-mediated dilatation technique}

The flow-mediated dilatation is obtained with the participant lying supine on a bed. This technique will be performed according to recently published consensus guidelines. ${ }^{16}$ The right arm will be placed in a specially designed supporting cradle and a blood pressure cuff will be placed around the forearm, with the edge of the cuff $5 \mathrm{~cm}$ distal to the medial epicondyle. A three-point electrocardiogram (both shoulders and left hip) connected to the ultrasound machine will allow display of the electrocardiographic trace on the ultrasound image. An ultrasound system (SonoSite M-Turbo, SonoSite Inc, Bothell, WA, USA) with a high resolution linear array transducer (SonoSite HLF38x) and a broadband frequency range of 6-13 $\mathrm{mHz}$ will be used to obtain high resolution images of the brachial artery. In particular, the transducer position will be adjusted until both the near and far arterial walls are well defined, the image is as free as possible of artifactual echoes, and the image size and gain are optimized. The transducer will be placed in a specially designed stereotactic stand that allows free movement while locating the brachial artery and then fixation of the transducer in any required position on the upper arm to allow constant imaging of the brachial artery over a prolonged period of time. The position of the transducer relative to the medial epicondyle will be recorded to ensure that the same part of the artery is imaged at the follow-up study.

A 30-second cine clip will be obtained prior to cuff inflation to allow a preinflation brachial artery diameter to be obtained. The cuff will then be inflated to $250 \mathrm{mmHg}$ to achieve total brachial artery occlusion. After cuff deflation, 190 seconds of assessment of the brachial artery will be obtained in the form of three $\times 60$-second clips with no more than five seconds between clips (five seconds are required for saving and starting the next clip), allowing almost continuous recording. Maximal dilatory response is expected to occur during this time, and the brachial artery diameter will be measured at the point of maximal dilatation. ${ }^{28}$

The cine clips will be transferred to the hospital Picture Archiving and Communication System (GE Centricity 3.0.3, GE Healthcare Integrated IT Solutions, Barrington, IL, USA) for permanent storage using the Digital Imaging and Communications in Medicine 3.0 standard format. These files will then be extracted from the Picture Archiving and Communication System and transferred to a computer 
workstation for analysis. Specific automated software (Brachial Artery Analyzer, MIA-LLC, Coralville, IA, USA) will be used to measure the brachial artery diameter before occlusion and continuously throughout the post-occlusion period for a total of 190 seconds. This software utilizes edge detection and wall tracking algorithms to measure the luminal diameter within a specified region of interest automatically. This technology has been shown to improve the validity of flow-mediated dilatation measurements compared with manual techniques. ${ }^{16}$ All measurements will be obtained in peak systole (using the displayed electrocardiographic trace) and will be the average of three measurements obtained from different cardiac cycles, all within five seconds of each other. A pre-occlusion diameter will be obtained, and a diameter will be obtained at the point of maximal dilatation during the postocclusion period, as identified by the tracking software.

Flow-mediated dilatation will be calculated by obtaining the percentage increase in diameter of the brachial artery and the absolute maximum diameter. The maximum percentage increase will be calculated from the pre-occlusion diameter and the maximum post-occlusion diameter, as per standard flow-mediated dilatation technique. ${ }^{16,20}$ The time from cuff deflation to maximum diameter will also be recorded.

\section{Laboratory analysis}

Following recruitment into the study, venipuncture will be performed at baseline and at 6 and 12 weeks. To assess the level of inflammation, fasting blood samples will be obtained and analyzed for C-reactive protein, absolute neutrophil, and lymphocyte values. Tumor necrosis factor- $\alpha$, interleukinIL-1 $\beta$, interleukin- 6 , thromboxane, and prostacyclin levels will be obtained using enzyme-linked immunosorbent assays. To assess lipid status, fasting blood samples will be obtained and analyzed for total cholesterol, high-density lipoprotein, low-density lipoprotein, and triglycerides. Analysis of fatty acid composition in the erythrocyte membrane will be measured to evaluate adherence with the fish oil intervention. Hyperhomocysteinemia is a commonly overlooked risk factor for atherosclerotic disease, and fish oil has been shown to lower homocysteine levels. ${ }^{29}$ For this reason, we will also assess fasting homocysteine levels. Nitric oxide levels are reduced in patients with endothelial dysfunction, so can act as a surrogate biochemical marker for endothelial function, ${ }^{10,11,16,20}$ but are rapidly degraded. More stable end products, ie, nitrite and nitrate ions, can be used to quantify nitric oxide. ${ }^{30}$ To achieve this, we will analyze serum samples using a microelectrode system with a sophisticated ion analyzer selective for nitrite and nitrate ions (ArrowStraight ${ }^{\mathrm{TM}}$ nitric oxide measurement system, Lazar Research Laboratories, Los Angeles, CA, USA).

\section{Assessment of background diet and physical activity}

At baseline and 12 weeks, participants will be required to complete two surveys, ie, the Australian Cancer Council Food Frequency Questionnaire and the International Physical Activity Questionnaire. The Australian Cancer Council Food Frequency Questionnaire ${ }^{31}$ is a well validated, 74-item semiquantitative questionnaire. The questions cover all food groups and beverages and provide participants with an opportunity to estimate portion sizes using illustrations. The questionnaires will be analyzed by the Australian Cancer Council, with estimates of nutrient intakes obtained using the NUTTAB95 database. The International Physical Activity Questionnaire to be administered is the Long Last 7 Days self-administered version, which consists of five activity domains, including job-related physical activity, physical activity related to transportation, physical activity related to housework, house maintenance, and caring for the family, physical activity related to recreation, sport, and leisure time, and time spent sitting. The maximum number of items is 27 and the time required to complete the test is approximately 10 minutes. The International Physical Activity Questionnaire has been tested extensively for validity and reliability in many settings and languages, and has been deemed suitable for determining participation in physical activity. ${ }^{32}$

\section{Statistical analysis}

All statistical analyses will be performed using Statistical Package for Social Sciences for Windows version 18 (SPSS Inc, Chicago, IL, USA). The primary analysis of this study will be undertaken using intention to treat principles. Central tendency and distribution will be determined and the data presented as appropriate. Independent samples $t$-tests, Mann-Whitney $U$ tests, and Chi-square tests of association will be used to compare the groups at baseline. To determine differences between the groups at the primary endpoint (12 weeks), analysis of covariance (main effect and interaction effect for baseline hyperemic index $<1.67$ versus hyperemic index 1.67-2.07) or logistic regression will be used, with models adjusted for potential confounders, including baseline hyperemic index.

\section{Power calculations}

Based on clinical expertise and the limited available data, we expect endothelial function of asymptomatic offspring 
of PAD patients to have a mean EndoPAT index of approximately $1.55 \pm 0.55$. With only a $10 \%$ improvement in EndoPAT index following administration of $1.5 \mathrm{~g}$ EPA and $1 \mathrm{~g}$ DHA for 12 weeks, we estimate that a sample size of 44 participants per group will be required (power $80 \%$, alpha $=0.05)$. To allow for attrition, 100 participants will be recruited (50 per group).

\section{Discussion}

To the best of our knowledge, this study is the first to examine the effectiveness of a moderate-dose fish oil intervention in reversing endothelial dysfunction in asymptomatic offspring of patients with PAD. Endothelial dysfunction can precede established PAD by decades. Therefore, an opportunity exists to provide high-risk asymptomatic offspring of these patients with a method of risk stratification, with the view that early detection of endothelial dysfunction using noninvasive hyperemia-peripheral artery tonometry index technology has the potential to alter clinical outcomes significantly. This is particularly relevant when we consider the reversibility of endothelial dysfunction even with simple lifestyle modifications, such as exercise or smoking cessation.

Fish oil is a cheap and readily available dietary supplement that may help complement such lifestyle modifications. The polyunsaturated fatty acids in fish oil have been demonstrated to not only improve vascular function in the setting of atherosclerotic disease, ${ }^{23}$ but also protect against cognitive decline $^{33}$ and reduce the risk of certain cancers, including breast, prostate and colon cancer. ${ }^{34-36}$ When presented to patients, these potential benefits may act as a tool to aid compliance, considering the offensive taste that is regularly reported in association with fish oil.

The status of fish oil as a natural supplement to endogenous substrates means it does not have the potential side effects associated with synthetic vasoactive pharmacological medications. While occasional gastrointestinal irritation has been attributed to fish oil, this is insignificant when compared with the muscle cramps, altered liver function tests, and electrolyte disturbances which are common following the onset of therapy with statins and antihypertensive medication.

There are no longitudinal studies documenting the rate of progression from endothelial dysfunction to PAD, largely because techniques to assess endothelial function are relatively new. However, when we consider that $10 \%-25 \%$ of the population over the age of 55 years have some degree of PAD,${ }^{1}$ the likelihood of patients with endothelial dysfunction progressing to PAD is significant. Together with the corresponding 2-3-fold increased risk of ischemic heart disease and stroke, the resources and costs to the health care system for the ongoing management of these patients is extreme and likely to worsen as the population continues to age. Therefore, the screening/risk stratification method and subsequent intervention that we propose in this trial has the potential to impact positively on health economics.

\section{Limitations}

Reactive hyperemia-peripheral artery tonometry index testing is a relatively new technique that is being re-evaluated on an ongoing basis in an attempt to ensure it has the characteristics to make it an optimal screening test. Currently, a value $<1.67$ has been proposed to represent established coronary endothelial dysfunction with a specificity of $77 \%$ and a sensitivity of $82 \% .{ }^{20}$ The value of $<2.07$ used in our study represents those deemed to be at high risk of progression to endothelial dysfunction. However, there is only weak evidence to support this, and work is continuing to improve the accuracy of risk stratification. Therefore, it is possible that our study may be either over-inclusive or under-inclusive rather than truly representative of the population of interest. The dose and duration of fish oil administration in this study is also worthy of acknowledgment. In an unpublished review undertaken by the authors, it was determined that in patients with established PAD, fish oil interventions have been administered from 4 weeks up to 2 years and at omega- 3 doses largely within 1-3 g daily, with inconsistent findings in terms of the magnitude and direction of the effect on clinically meaningful outcomes, such as pain-free walking distance. These findings in addition to the suggestion that it takes at least 10-12 weeks for EPA and DHA to incorporate into cell membranes effectively ${ }^{22}$ informed the intervention to be administered in this study rather than any solid evidence from the target population.

\section{Acknowledgments}

The authors would like to acknowledge Sheralee Sandison for her assistance in distribution of flyers for recruitment of participants and the administrative staff of Flinders University Nutrition and Dietetics for responding to telephone enquiries and general administrative duties associated with conduct of the research project. Partial funding for this research was received through the Flinders University Faculty of Health Sciences seeding grants.

\section{Disclosure}

The authors declare that they have no competing interests in this work. 


\section{References}

1. Norman PE, Eikelboom JW, Hankey GJ. Peripheral arterial disease: prognostic significance and prevention of atherothrombotic complications. Med J Aust. 2004;181(3):150-154.

2. National Heart Foundation of Australia and Access Economics. The shifting burden of cardiovascular disease in Australia. Report prepared by Access Economics for the National Heart Foundation of Australia. Canberra, Australia: Access Economics; 2005. Available from: http:// www.heartfoundation.org.au/sitecollectiondocuments/hf-shifting burden-cvd-accecons-2005-may.Pdf. Accessed May 5, 2013.

3. Australian Bureau of Statistics. Cardiovascular Disease in Australia: A Snapshot. Catalog number 4821.0.55.001. Canberra, Australia: Australian Bureau of Statistics; 2001.

4. Dalager-Pedersen S, Ravn HB, Falk B. Atherosclerosis and acute coronary events. Am J Cardiol. 1998;82(10B):37T-40T.

5. Australian Institute of Health and Welfare. Heart, stroke, and vascular diseases - Australian facts. Catalog number CVD 27. Canberra, Australia: Australian Institute of Health and Welfare; 2004. Available from: http://www.aihw.gov.au/WorkArea/DownloadAsset. aspx?id=6442454949. Accessed May 5, 2013.

6. Australian Institute of Health and Welfare. Peripheral vascular disease in Australia. Available from: http://www.aihw.gov.au/cvd/peripheral_ vascular_disease.cfm. Accessed November 11, 2011.

7. Australian Bureau of Statistics. Cardiovascular Disease in Australia: A snapshot, 2004-2005. Catalog number 4821.0.55.001. Canberra, Australia: Australian Bureau of Statistics; 2006.

8. Gokce N, Keaney JF Jr, Hunter LM, Watkins MT, Menzoian JO, Vita JA. Risk stratification for postoperative cardiovascular events via noninvasive assessment of endothelial function: a prospective study. Circulation. 2002;105(13):1567-1572.

9. Bacon SL, Lavoie KL, Arsenault A, et al. The Research on Endothelial function in Women And men at Risk for cardiovascular Disease (REWARD) study: methodology. BMC Cardiovasc Disord. 2011;11:50.

10. Celermajer DS, Sorensen KE, Gooch VM, et al. Non-invasive detection of endothelial dysfunction in children and adults at risk of atherosclerosis. Lancet. 1992;340(8828):1111-1115.

11. Laurent S, Lacolley P, Brunel P, Laloux B, Pannier B, Safar M. Flowdependent vasodilation of brachial artery in essential hypertension. Am J Physiol. 1990;258(4 Pt 2):H1004-H1011.

12. Kuvin JT, Mammen A, Mooney P, Alsheikh-Ali AA, Karas RH. Assessment of peripheral vascular endothelial function in the ambulatory setting. Vasc Med. 2007;12(1):13-16.

13. Inaba Y, Chen JA, Bergmann SR. Prediction of future cardiovascular outcomes by flow-mediated vasodilatation of brachial artery: a metaanalysis. Int J Cardiovasc Imaging. 2010;26(6):631-640.

14. de Jongh S, Lilien MR, op't Roodt J, Stroes ES, Bakker HD, Kastelein JJ. Early statin therapy restores endothelial function in children with familial hypercholesterolemia. J Am Coll Cardiol. 2002;40(12):2117-2121.

15. Antony I, Lerebours G, Nitenberg A. Angiotensin-converting enzyme inhibition restores flow-dependent and cold pressor test-induced dilations in coronary arteries of hypertensive patients. Circulation. 1996;94(12):3115-3122.

16. Thijssen DHJ, Black MA, Pyke KE, et al. Assessment of flow-mediated dilation in humans: a methodological and physiological guideline. Am J Physiol Heart Circ Physiol. 2011;300(1):H2-H12.

17. Ganz P, Vita JA. Testing endothelial vasomotor function. Nitric oxide, a multipotent molecule. Circulation. 2003;108(17):2049-2053.

18. Drexler H, Hornig B. Endothelial dysfunction in human disease. $J \mathrm{Mol}$ Cell Cardiol. 1999;31(1):51-60.
19. Costa C, Virag R. The endothelial-erectile dysfunction connection: an essential update. $J$ Sex Med. 2009;6(9):2390-2404.

20. Bonetti PO, Pumper GM, Higano ST, Holmes DR Jr, Kuvin JT, Lerman A. Noninvasive identification of patients with early coronary atherosclerosis by assessment of digital reactive hyperemia. $\mathrm{J} \mathrm{Am} \mathrm{Coll}$ Cardiol. 2004;44(11):2137-2141.

21. Zintzaras E, Zdoukopoulos N. A Field synopsis and meta-analysis of genetic association studies in peripheral arterial disease: the CUMAGAS-PAD database. Am J Epidemiol. 2009;170(1):1-11.

22. Carrero JJ, Grimble RF. Does nutrition have a role in peripheral vascular disease? Br J Nutr. 2006;95(2):217-229.

23. Schiano V, Laurenzano E, Brevetti G, et al. Omega-3 polyunsaturated fatty acid in peripheral arterial disease: effect on lipid pattern, disease severity, inflammation profile, and endothelial function. Clin Nutr. 2008;27(2):241-247.

24. Greenland P, Abrams J, Aurigemma GP, et al. Prevention Conference V: beyond secondary prevention: identifying the high-risk patient for primary prevention: noninvasive tests of atherosclerotic burden: Writing Group III. Circulation. 2000;101(1):E16-E22.

25. Lucas M, Asselin G, Merrette C, Poulin M, Dodin S. Validation of an FFQ for evaluation of EPA and DHA. Public Health Nutr. 2008;12(10): $1783-1790$

26. Goodfellow J, Bellamy MF, Ramsey MW, Jones CJH, Lewis MJ. Dietary supplementation with marine omega-3 fatty acids improve systemic large artery endothelial function in subjects with hypercholesterolemia. J Am Coll Cardiol. 2000;35(2):265-270.

27. Cleland LG, Caughey GE, James MJ, Proudman SM. Reduction of cardiovascular risk factors with longterm fish oil treatment in early rheumatoid arthritis. J Rheumatol. 2006;33(10):1973-1979.

28. Coretti MC, Anderson TJ, Benjamin EJ, et al. Guidelines for the ultrasound assessment of endothelial-dependent flow-mediated vasodilation of the brachial artery: a report of the International Brachial Artery Reactivity Task Force. J Am Coll Cardiol. 2002;39(6): 257-265.

29. Olszewski AJ, McCully KS. Fish oil decreases serum homocysteine in hyperlipemic men. Coron Artery Dis. 1993;4(1):53-60.

30. Kleinbongard P, Dejam A, Lauer T, et al. Plasma nitrite reflects constitutive nitric oxide synthase activity in mammals. Free Radic Biol Med. 2003;35(7):790-796.

31. Giles GG, Ireland PD. Dietary Questionnaire for Epidemiological Studies (Version 2). Melbourne, Australia: The Cancer Council Victoria; 1996.

32. Booth ML. Assessment of physical activity: an international perspective. Res Q Exerc Sport. 2000;71(2):s114-s120.

33. Lukiw WJ, Cui JG, Marcheselli VL, et al. A role for docosahexaenoic acid-derived neuroprotectin D1 in neural cell survival and Alzheimer disease. J Clin Invest. 2005;115(10):2774-2783.

34. de Deckere EA. Possible beneficial effect of fish and fish n-3 polyunsaturated fatty acids in breast and colorectal cancer. Eur J Cancer Prev. 1999;8(3):213-221.

35. Augustsson K, Michaud DS, Rimm EB, et al. A prospective study of intake of fish and marine fatty acids and prostate cancer. Cancer Epidemiol Biomarkers Prev. 2003;12(1):64-67.

36. Caygill CP, Hill MJ. Fish, n-3 fatty acids and human colorectal and breast cancer mortality. Eur J Cancer Prev. 1995;4(4):329-332. 
Open Access Journal of Clinical Trials

Dovepress

\section{Publish your work in this journal}

The Open Access Journal of Clinical Trials is an international, peerreviewed, open access journal publishing original research, reports, editorials, reviews and commentaries on all aspects of clinical tria design, management, legal, ethical and regulatory issues, case record form design, data collection, quality assurance and data auditing methodologies. The manuscript management system is completely online and includes a very quick and fair peer-review system, which is all easy to use. Visit http://www.dovepress.com/testimonials.php to read real quotes from published authors.

Submit your manuscript here: http://www.dovepress.com/open-access-journal-of-clinical-trials-journal 